\title{
ON CONSTRUCTION OF A LONG-TERM MOON'S THEORY
}

\author{
T.V. IVANOVA \\ Institute of Applied Astronomy of the Russian Academy of Sciences, \\ St.Petersburg,E-mail: itv@ita.spb.su
}

An analytical long-term theory of the motion of the Moon is constructed within the framework of the general planetary theory (Brumberg, 1995). A method, different from the one of (Ivanova, 1997) designated below as (*), for the determination of the perturbations depending on the eccentricities and inclinations of lunar and planetary orbits is used which allows to obtain the solution of the problem in the purely trigonometric form up to any order with respect to the small parameters.

The aim of this paper is to construct the long-term Lunar theory in the form consistent with the general planetary theory (Brumberg, 1995). For this purpose the Moon is considered as an additional planet in the field of eight major planets (Pluto being excluded). In the result the coordinates of the Moon may be represented by means of the power series in the evolutionary eccentric and oblique variables with trigonometric coefficients in mean longitudes of the Moon and the planets. The long-period perturbations are determined by solving a secular system in Laplacetype variables describing the secular motions of the lunar perigee and node and taking into account the secular planetary inequalities. This paper is a development of results obtained in $\left(^{*}\right)$. Unlike $\left(^{*}\right)$ the equations of the Moon's motion are described in the different form

$$
\begin{gathered}
\ddot{p}+2 \sqrt{-1} n \dot{p}-\frac{3}{2} n^{2}(p+q)+n^{2} \sum_{i=1}^{9}\left(K_{i} \delta p_{i}+L_{i} \delta q_{i}\right)=n^{2} P^{\prime}, \\
\ddot{w}+n^{2} w+n^{2} \sum_{i=1}^{9} M_{i} w_{i}=n^{2} W^{\prime} .
\end{gathered}
$$

Here the variables $p, q, w$ are introduced by $x+\sqrt{-1} y=\mathrm{a}(1-p) \exp \sqrt{-1} \lambda, q=$ $\bar{p}, z=\mathrm{a} w, w=\bar{w}$ instead of geocentric rectangular coordinates $\mathbf{r}=(x, y, z)$ and represent the small deviations from the plane circular lunar motion. The bar means a conjugate value. a, $n, \lambda$ are the semi-major axis, mean motion and longitude of the Moon, respectively. The heliocentric coordinates $\mathbf{r}_{\mathbf{i}}=\left(x_{i}, y_{i}, z_{i}\right)(i=1,2, \ldots, 8)$ of the principal planets are subjected to similar transformation. For the Moon the values both without any indexes and with index 9 are used. The right-hand members are expressed in the form

$$
\begin{array}{cc}
P^{\prime}=P-P^{(0)}+\sum_{i=1}^{9}\left(K_{i} \delta p_{i}+L_{i} \delta q_{i}\right), & W^{\prime}=W+\sum_{i=1}^{9} M_{i} w_{i}, \\
P=-1-\frac{1}{2} p-\frac{3}{2} q+\frac{2}{n^{2} \mathrm{a}^{2}} \frac{\partial U}{\partial q}, & W=w+\frac{1}{n^{2} \mathrm{a}^{2}} \frac{\partial U}{\partial w},
\end{array}
$$


$U$ being the force function. In accordance with the general planetary theory one has $p_{i}=p_{i}^{(0)}+\delta p_{i}$. The intermediary $p=p^{(0)}, w=0$ is a particular planar quasiperiodic solution of equations (1) with the the right-hand members $P=P^{(0)}$, $W=0$ provided that the major planets move in their intermediate orbits. This solution generalizes Hill's variational curve and includes all solar and planetary inequalities independent of eccentricities and inclinations of all bodies. It is built in the similar way as in (*). The coefficients $K_{i}, L_{i}, M_{i}$ are functions of the intermediate solution of the bodies under consideration. The perturbations $\delta p$ and $w$ depending on the eccentricities and inclinations of the planetary and lunar orbits are determined by different method than in $\left(^{*}\right)$ by means of separating linear terms in the eccentricities and the inclinations of all bodies from the right-hand members. The right-hand members $P$ and $W$ used in (*) contain the terms of first order with respect to $\delta p_{i}$ and $w_{i}$ while $P^{\prime}$ and $W^{\prime}$ start with the second degree terms of $\delta p_{i}$ and $w_{i}$. It guarantees the convergence of iterations. Finally, the solution for $\delta p$ and $w$ is sought in the form of power series

$$
\delta p=\sum p_{k l m n} \prod_{i=1}^{9} a_{i}^{k_{i}} \bar{a}_{i}^{l_{i}} b_{i}^{m_{i}} \bar{b}_{i}^{n_{i}}, \quad w=\sum w_{k l m n} \prod_{i=1}^{9} a_{i}^{k_{i}} \bar{a}_{i}^{l_{i}} b_{i}^{m_{i}} \bar{b}_{i}^{n_{i}}
$$

with the initial approximation

$$
\delta p=-\frac{1}{2} a_{9}+\frac{3}{2} \bar{a}_{9}+\sum_{i=1}^{9}\left(c_{i} a_{i}+d_{i} \bar{a}_{i}\right), \quad w=b_{9}+\bar{b}_{9}+\sum_{i=1}^{9}\left(f_{i} b_{i}+\bar{f}_{i} b_{i}\right),
$$

where $a_{i}$ and $b_{i}$ are the complex variables of Laplace-type proportional to the eccentricity and inclination of the body with number $i$, respectively. The functions $c_{i}, d_{i}, f_{i}$ depend on the intermediary of all bodies. The summation is performed over all non-negative values of 9 -indices $k, l, m, n$. The coefficients in (2) are quasi-periodic functions of time. The method of $\delta p$ and $w$ construction is based on the separation of the fast and slowly changing variables and on the Birkhoff normalization. All analytical calculations are performed by the specialized Poisson series processor PSP (Ivanova, 1996). This research is supported by the Russian Foundation of Fundamental Researches (grant No. 97-01-01176) and the Ministry of Science of Russia (Astronomy).

\section{References}

Brumberg V. A.: 1995, Analytical Techniques of Celestial Mechanics, Springer, Heidelberg. Ivanova, T. V.: 1996, "PSP: A New Poisson Series Processor", in: Dynamics, Ephemerides and Astrometry of the Solar System (eds. S.Ferraz-Mello, B.Morando and J.-E.Arlot), 283, Kluwer. Ivanova, T. V.: 1997, "A Trigonometric Solution of the Secular Lunar System Intended for Long-Term Theory of the Earth's Rotation", in: Journees 1997 "Systemes de Reference Spatio-Temporels" (eds. J. Vondrak and N. Capitaine), 99, Prague. 